\title{
Dynamic Snap-Through of Thin-Walled Structures by a Reduced Order Method
}

\author{
Adam Przekop* \\ National Institute of Aerospace, Hampton, VA 23666 \\ Stephen A. Rizzi ${ }^{\dagger}$ \\ NASA Langley Research Center, Hampton, VA 23681
}

\begin{abstract}
The goal of this investigation is to further develop nonlinear modal numerical simulation methods for application to geometrically nonlinear response of structures exposed to combined high intensity random pressure fluctuations and thermal loadings. The study is conducted on a flat aluminum beam, which permits a comparison of results obtained by a reduced-order analysis with those obtained from a numerically intensive simulation in physical degrees-of-freedom. A uniformly distributed thermal loading is first applied to investigate the dynamic instability associated with thermal buckling. A uniformly distributed random loading is added to investigate the combined thermal-acoustic response. In the latter case, three types of response characteristics are considered, namely: (i) small amplitude vibration around one of the two stable buckling equilibrium positions, (ii) intermittent snap-through response between the two equilibrium positions, and (iii) persistent snap-through response between the two equilibrium positions. For the reducedorder analysis, four categories of modal basis functions are identified including those having symmetric transverse (ST), anti-symmetric transverse (AT), symmetric in-plane (SI), and anti-symmetric in-plane (AI) displacements. The effect of basis selection on the quality of results is investigated for the dynamic thermal buckling and combined thermal-acoustic response. It is found that despite symmetric geometry, loading, and boundary conditions, the AT and SI modes must be included in the basis as they participate in the snap-through behavior.
\end{abstract}

\section{Nomenclature}

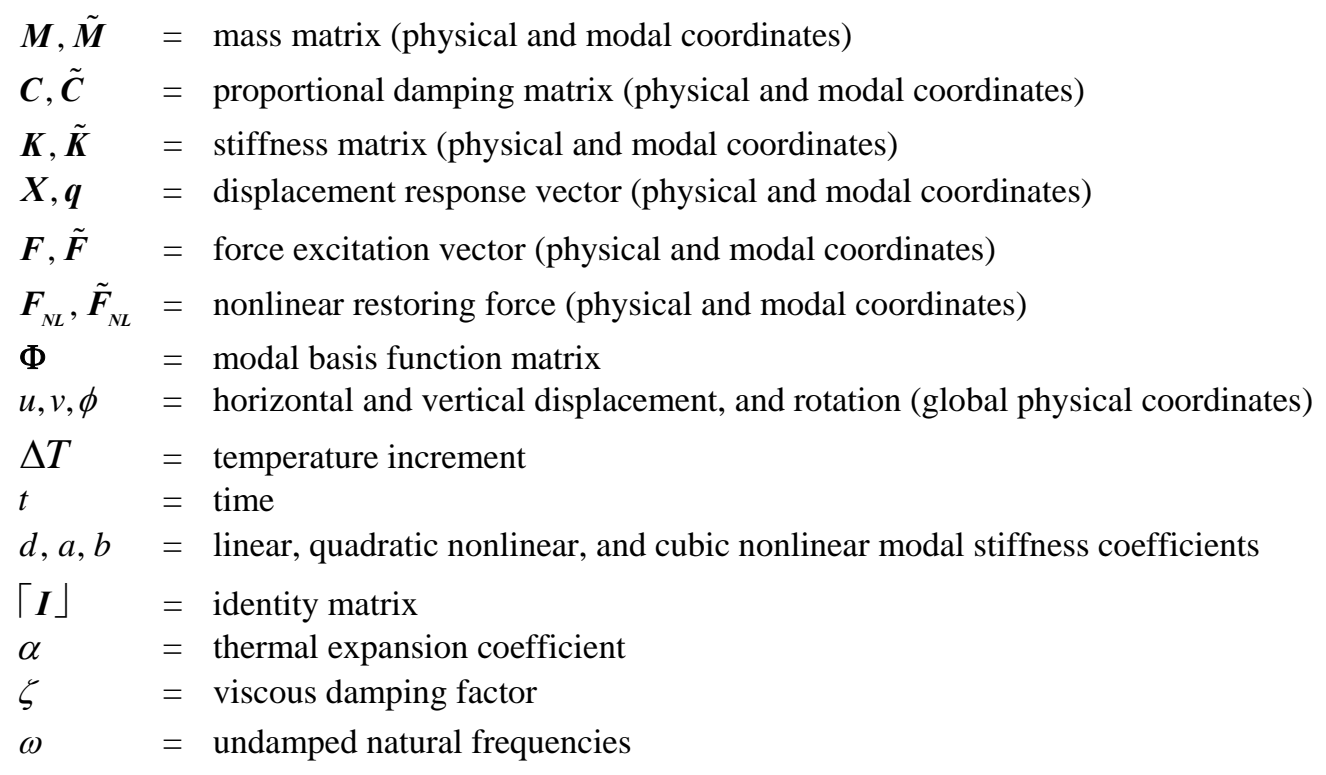

\footnotetext{
${ }^{*}$ Research Scientist, AIAA Senior Member.

${ }^{\dagger}$ Aerospace Engineer, Structural Acoustics Branch, AIAA Associate Fellow.
} 


\section{Introduction}

$\mathrm{D}$ RECT numerical simulation of nonlinear random response in physical degrees-of-freedom (DoF) is computationally intensive even for the simplest structures. Its use for design of high-cycle-fatigue tolerant aerospace vehicle structures, requiring long simulation times to obtain meaningful statistics, is considered impractical. Accordingly, much effort has been spent in recent years to develop accurate reduced-order analyses, such as finite-element-based nonlinear modal numerical simulation, which could be suitable for use in design environments.

Aerospace structures exposed to a high-intensity random acoustic loading are often also simultaneously exposed to an elevated thermal environment. Since both acoustic and thermal loadings can cause the structure to respond in a geometrically nonlinear fashion, an analysis technique that permits simultaneous loading is required, i.e. linear superposition of the acoustic and thermal response is not suitable. Under certain thermal-acoustic loading conditions, a dynamic instability will give rise to a snap-though response, ${ }^{1-3}$ which can significantly reduce fatigue life. The snap-through problem has previously been investigated using reduced-order analyses with both closedform $^{4-6}$ and finite element $(\mathrm{FE})^{7-9}$ solutions. Reduced-order FE analysis can be further classified into so-called direct $^{7,8}$ and indirect ${ }^{9}$ stiffness evaluation procedure approaches. For both direct and indirect approaches, the system is first transformed to a reduced set of coupled nonlinear equations, which are solved via numerical integration. Since the eventual application is the analysis and design of practical structures, this paper focuses on developments associated with the indirect approach, which has been implemented for use with commercial finite element codes. ${ }^{9-}$

11 The previous work ${ }^{9}$ using the indirect procedure modeled the dynamic snap-through response with a single transverse mode. However, it has been subsequently shown that a significant improvement can be obtained using a basis consisting of both low-frequency transverse-dominated modes and high-frequency in-plane-dominated modes. ${ }^{11,12}$ The present work concentrates on selecting such a basis so that both the transverse and in-plane dynamic behaviors of the system can be accurately modeled.

A clamped-clamped aluminum beam is considered in this work to allow reduced-order analysis results to be compared with a numerically intensive simulation in physical DoF. The dynamic thermal buckling problem is first studied by applying a uniformly distributed, positive temperature increment. The combined thermal-acoustic loading is subsequently investigated through the addition of uniformly distributed acoustic loadings of different intensities. Several response characteristics are investigated including: (i) small amplitude vibration around one of two stable, buckled equilibrium positions, (ii) intermittent snap-through response between the two buckled equilibrium positions, and (iii) persistent snap-through response between the two buckled equilibrium positions. In each case, the reduced-order analysis is performed with two different sets of basis functions, and results are compared with those obtained by numerical simulation in physical DoF.

\section{Reduced-Order Numerical Simulation}

The reduced-order analysis is first presented as it is used to study the response to both thermal and thermalacoustic loadings. Similarities between the direct and indirect stiffness evaluation methods are discussed.

\section{A. Modal Coordinate Transformation}

In the direct stiffness evaluation approach, the equations of motion for a nonlinear system subjected to a change in temperature can be expressed in the form ${ }^{7,8,13}$

$$
\boldsymbol{M} \ddot{\boldsymbol{X}}(t)+\boldsymbol{C} \dot{\boldsymbol{X}}(t)+\left[K_{\boldsymbol{L}}-K_{\Delta T}(\Delta T)+K_{1}(\boldsymbol{X}(t))+K_{2}(\boldsymbol{X}(t), \boldsymbol{X}(t))\right] \boldsymbol{X}(t)=\boldsymbol{F}(t)+\boldsymbol{F}_{\Delta \boldsymbol{T}}
$$

where $\boldsymbol{M}$ is the mass matrix, $\boldsymbol{C}$ is the mass proportional damping matrix (no temperature dependence is assumed), and $K_{L}, K_{1}$, and $K_{2}$ are the linear, quadratic, and cubic stiffness matrices, respectively. $\boldsymbol{X}$ is the displacement response vector and $\boldsymbol{F}$ is the force excitation vector. The thermal effect is present on both sides of Eq. (1); as a change in the linear stiffness matrix $K_{\Delta T}(\Delta T)$ on the left-hand-side, and as a thermal force vector $\boldsymbol{F}_{\Delta T}$ on the righthand-side.

In the indirect stiffness evaluation approach, the equation of motion is written in the form

$$
\boldsymbol{M} \ddot{\boldsymbol{X}}(t)+\boldsymbol{C} \dot{\boldsymbol{X}}(t)+\boldsymbol{F}_{N L}(\boldsymbol{X}(t), \Delta T)=\boldsymbol{F}(t)
$$


Here, the thermal effect is represented entirely on the left-hand-side of the equation in the nonlinear restoring force $\boldsymbol{F}_{N L}$, which also contains the linear, quadratic and cubic stiffness terms. Comparing Eqs. (1) and (2), a relationship between direct and indirect formulations is established, namely,

$$
\boldsymbol{F}_{N L}(\boldsymbol{X}(t), \Delta T)=\left[K_{L}-K_{\Delta T}(\Delta T)+K_{1}(\boldsymbol{X}(t))+K_{2}(\boldsymbol{X}(t), \boldsymbol{X}(t))\right] \boldsymbol{X}(t)-\boldsymbol{F}_{\Delta \boldsymbol{T}}
$$

Continuing with the indirect approach, a set of coupled modal equations with reduced DoF is obtained by applying the modal coordinate transformation $\boldsymbol{X}=\boldsymbol{\Phi} \boldsymbol{q}$ to Eq. (2), where $\boldsymbol{q}$ is the modal displacement response vector. In this study, the set of modal basis functions, $\Phi$, is formed from the linear eigenvalue problem using only that part of the restoring force associated with the linear stiffness, without the effect of temperature. These are sometimes referred to as "cold modes." Generally, a small set of $L$ basis functions is included resulting in a modal equation of motion that takes the form

$$
\tilde{\boldsymbol{M}} \ddot{\boldsymbol{q}}(t)+\tilde{\boldsymbol{C}} \dot{\boldsymbol{q}}(t)+\tilde{\boldsymbol{F}}_{N L}\left(q_{1}(t), q_{2}(t), \ldots, q_{L}(t), \Delta T\right)=\tilde{\boldsymbol{F}}(t)
$$

The tilde superscript represents modal quantities, and

$$
\tilde{\boldsymbol{M}}=\boldsymbol{\Phi}^{T} \boldsymbol{M} \boldsymbol{\Phi}=\lceil\boldsymbol{I}\rfloor \quad \tilde{\boldsymbol{C}}=\boldsymbol{\Phi}^{T} \boldsymbol{C} \boldsymbol{\Phi}=\left\lceil 2 \zeta_{r} \omega_{r}\right\rfloor \quad \tilde{\boldsymbol{F}}_{N L}=\boldsymbol{\Phi}^{T} \boldsymbol{F}_{N L} \quad \tilde{\boldsymbol{F}}=\boldsymbol{\Phi}^{T} \boldsymbol{F} .
$$

\section{B. Indirect Stiffness Evaluation Method}

The previously developed ${ }^{9-11}$ indirect stiffness evaluation procedure is used. To summarize, the r-th element of the nonlinear restoring force vector in Eq. (4) can be formed by computing

$$
\tilde{F}_{N L}^{r}\left(q_{1}, q_{2}, \ldots, q_{L}, \Delta T\right)=\sum_{j=1}^{L} d_{j}^{r}(\Delta T) q_{j}+\sum_{j=1}^{L} \sum_{k=j}^{L} a_{j k}^{r} q_{j} q_{k}+\sum_{j=1}^{L} \sum_{k=j}^{L} \sum_{l=k}^{L} b_{j k l}^{r} q_{j} q_{k} q_{l} \quad r=1,2, \ldots, L
$$

where $d, a$, and $b$ are the linear, quadratic nonlinear, and cubic nonlinear modal stiffness coefficients, respectively. The indirect stiffness evaluation procedure reduces the problem of determining the nonlinear modal stiffness to a series of static nonlinear problems with prescribed displacement fields and, if required, temperatures. The prescribed displacement fields are formed from combinations of modes in the basis. ${ }^{9-11}$ Once the resulting nonlinear forces are determined, the nonlinear modal stiffness coefficients may be found by solution of a simple algebraic system of equations. Note that the thermal loading can have an arbitrary spatial and through-the-thickness distribution, as long as such a distribution is supported by a commercial FE code used to compute the nonlinear restoring forces.

\section{Implementation}

The program RANSTEP was used to perform the reduced-order analysis with the indirect stiffness evaluation procedure. In particular, the RANSTEP implementation for MSC.NASTRAN was used because of successful application to similar problems. ${ }^{11}$ The implementation consists of several steps. The linear eigenvectors are first obtained from a normal modes analysis (Solution 103). The required displacement fields are then formed as a summation of selected and appropriately scaled basis functions. Next, a series of static nonlinear solutions (Solution 106) are performed at a prescribed elevated temperature to obtain the corresponding restoring forces. Based on these forces, the modal stiffness coefficients are evaluated. The resulting coupled system of equations, Eq. (4), is numerically integrated using the fourth order Runge-Kutta scheme to obtain a modal displacement time history. An inverse modal transformation allows the physical displacement to be computed.

\section{Finite Element Model}

The beam under analysis measured 18-in. $\mathrm{x} 1$-in. $\mathrm{x}$ 0.09-in (length $\mathrm{x}$ width $\mathrm{x}$ thickness). The FE model consisted of 144 CBEAM beam elements, each measuring 0.125 -in. in length. Clamped boundary conditions were applied at both ends of the beam by specifying zero displacement and rotation. The following material properties were used:

$$
E=10.6 \times 10^{6} \mathrm{psi}, \quad G=4.0 \times 10^{6} \mathrm{psi}, \quad \rho=2.588 \times 10^{-4} \frac{\mathrm{lb}_{f}-\mathrm{s}^{2}}{i \mathrm{i}^{4}}, \quad \alpha=12.4 \frac{\mu-i n / i n}{{ }^{\circ} \mathrm{F}} .
$$

In the analyses that follow, mass proportional damping corresponding to critical damping of $2.0 \%$ for the fundamental mode (at $57.8 \mathrm{~Hz}$ ) was specified.

Displacement results presented later in the paper are in the global coordinate system, which has its origin at the left clamped end of the beam. The $x$-axis coincides with the beam mid-surface and stretches along its span, with the positive $y$-axis pointing upwards. Thus, the in-plane $(u)$ displacement is in the direction of the $x$-axis, and the transverse $(v)$ component in the direction of the $y$-axis. 


\section{E. Modal Basis Classification and Selection}

It is helpful to categorize the linear eigenvectors that constitute the modal basis by their spatial distribution and by the dominant DoF, e.g. transverse displacement. For the flat, isotropic and symmetric beam structure considered herein, four categories are defined. Those having symmetric transverse displacements are subsequently referred to as symmetric transverse (ST) modes, while those having anti-symmetric transverse displacements are referred to as anti-symmetric transverse (AT) modes. Additionally, those having symmetric in-plane displacements are referred to as symmetric in-plane (SI) modes, while those having anti-symmetric in-plane displacements are referred to as antisymmetric in-plane (AI) modes. Due to the absence of linear coupling between transverse and in-plane modes for the structure considered, both ST and AT modes have numerical zero in-plane displacement components $(u)$. Similarly, both SI and AI modes have numerical zero transverse displacement components $(v)$. Non-zero rotational DoF $(\phi)$ are present only in the transverse ST and AT modes. ST modes have an anti-symmetric rotation distribution along the beam span, and AT modes have a symmetric rotation distribution along the beam span. The lowest mode of each of the four categories discussed above is presented in Figure 1. The listing of the first six modes of each category is provided in Table 1. It is seen that the transverse ST and AT modes have lower natural frequencies than the in-plane SI and AI modes.

Table 1: Classification of selected eigenvectors.

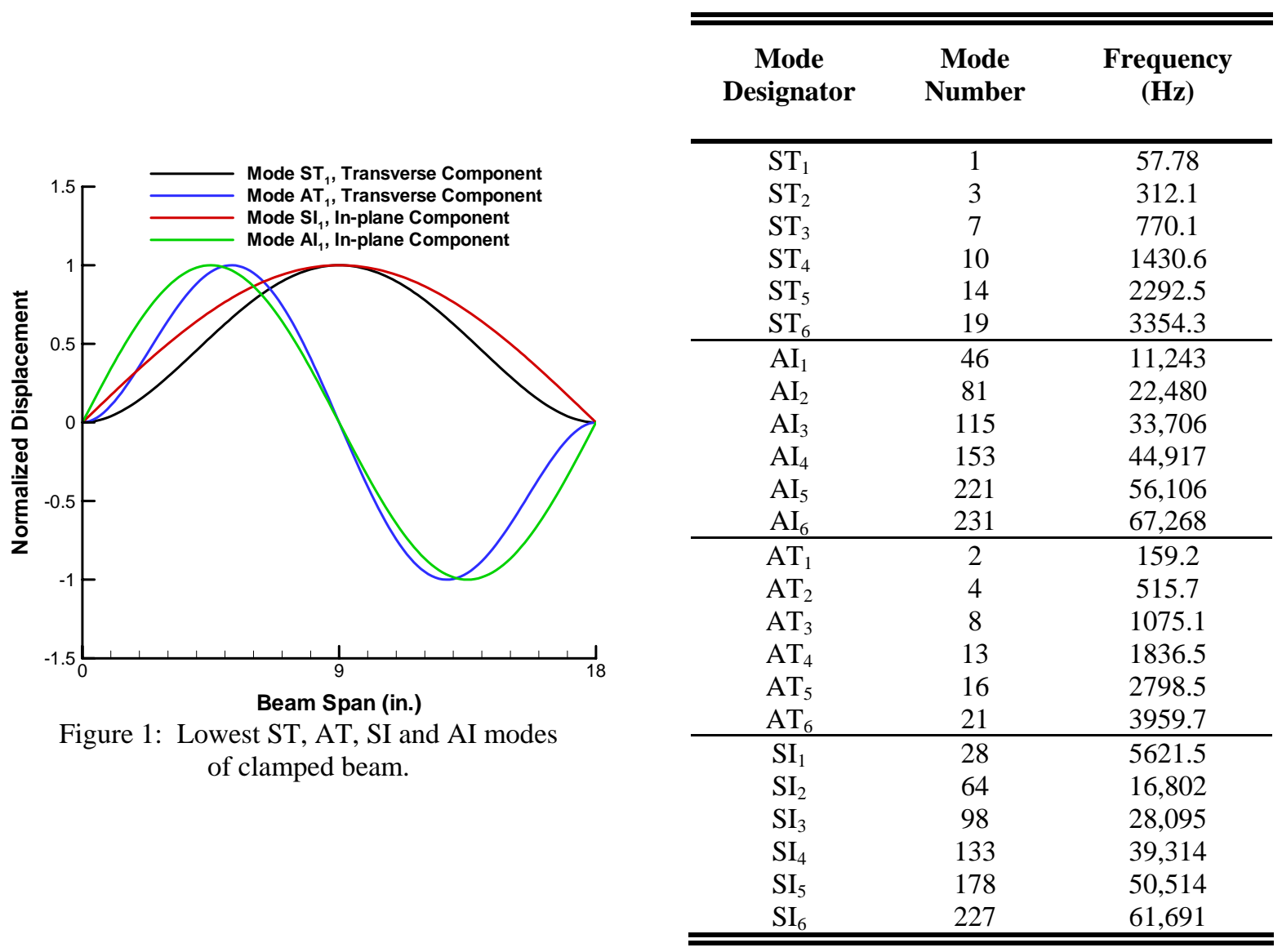

The most critical task in the reduced-order analysis is the selection of the modal basis, as it dictates the accuracy of the reduced-order solution. It may be intuitive to select a modal basis using characteristics of structural and loading symmetry. For example, for a symmetric planar structure under symmetric transverse loading, a modal basis consisting of only ST modes may suffice. While such intuition may be useful for a linear analysis, it has been demonstrated for both flat ${ }^{11}$ and curved ${ }^{12}$ structures that such a rationale will lead to an inadequate basis selection for the nonlinear problem. This is due to coupling in the quadratic and cubic stiffness terms. Therefore, some additional guidance in the form of experimental data, theoretical or numerical analysis, or past reduced-order modeling experience is beneficial.

For the beam considered, several factors affected the modal basis selection used in this study. The first factor was earlier experience gained from a reduced-order analysis of symmetric planar structures under symmetric 
transverse loadings at room temperature $(\Delta T=0)$ conditions. ${ }^{11}$ In this case, a combination of low-frequency ST modes and high-frequency AI modes was found to compare very well with a numerical simulation in physical DoF. From the study of shallowly curved structures, ${ }^{12}$ the additional inclusion of AT and SI modes was required to capture the in-plane behavior associated with autoparametric resonance. The geometry of the curved structure is similar to that of the thermally post-buckled beam, and hence the inclusion of AT and SI modes for the present problem is likely beneficial. Finally, through observations made using results to be subsequently presented from the numerical simulation analysis in physical DoF, significant in-plane motion of the center node was found to accompany the snap-through response, further substantiating the need for SI modes in the modal basis.

Thus, the modal basis chosen for this study consisted of all four types of modes. Based on the frequency range of the excitation, the six lowest modes of each four types were used to establish the modal basis. In the discussion that follows, this set will be referred to as 24-mode basis. To explore the impact of selecting an insufficient basis on the quality of the reduced-order results, a truncated basis was assembled from the six lowest ST modes and the six lowest AI modes. This basis lacked the SI modes necessary to capture the in-plane motion at the beam center. In the following, it will be referred to as 12-mode basis.

\section{F. Modal Stiffness Coefficients}

The behavior of modal stiffness coefficients as a function of applied temperature increment was examined and warrants a discussion. For the purpose of this investigation, modal stiffness coefficients were computed at room temperature, and at a uniformly distributed $\Delta T$ of $35^{\circ} \mathrm{F}$ and $70^{\circ} \mathrm{F}$. There was no thermal gradient through the thickness. Since the critical buckling temperature $\Delta T_{C R}$ of the beam was found to be $6.6^{\circ} \mathrm{F}$, the two elevated temperature cases corresponded to approximately 5.3 and 10.6 times $\Delta T_{C R}$, respectively. Both the 12- and 24-mode bases were used.

Examination of the nonlinear quadratic and cubic modal stiffness matrices revealed that they were not affected by the temperature change. This observation is in agreement with the classic FE formulation, where the nonlinear stiffness matrices are only a function of displacement, regardless of how this displacement was induced. ${ }^{7,8,13}$ The linear modal stiffness coefficients however were found to be strongly dependent on the temperature change. For the room temperature condition, the linear modal stiffness matrix is uncoupled. The linear stiffness coefficients are the positive eigenvalues and equal to the square of the natural frequencies of the system. Further, since the matrix is diagonal, the linear modal stiffness does not contribute to the overall coupling of transverse and in-plane modes. At elevated temperatures, some of the off-diagonal stiffness terms become significant, resulting in a coupled linear modal stiffness matrix. For both temperatures and bases considered, only the portion of the linear matrix corresponding to the low-frequency transverse modes (ST in the 12-mode basis, and ST and AT in the 24-mode basis) was altered. The off-diagonal terms were symmetric. These observations are also consistent with the previous classic FE development. ${ }^{13}$ Moreover, in the case of the 12-mode basis, the low-frequency portion of the linear stiffness was fully populated, effectively coupling all ST modes included in the basis. In the case of the 24mode basis, the coupling between ST modes was large, and the coupling between AT modes was large, but no linear cross-coupling occurred between the ST and AT modes. These observations are illustrated in Table 2 with excerpts from the linear modal stiffness matrices computed with the 24-mode basis at different thermal loadings.

Table 2: Selected modal linear stiffness $d_{i j}$ coefficients $\left(\mathrm{x} 10^{6}\right)$ for the 24-mode basis.

\begin{tabular}{|c|c|c|c|c|}
\hline$\Delta \boldsymbol{T}=\mathbf{0}^{\circ} \mathbf{F}$ & $\mathbf{S T}_{\mathbf{1}}$ & $\mathbf{A T}_{\mathbf{1}}$ & $\mathbf{S T}_{\mathbf{2}}$ & $\mathbf{A T}_{\mathbf{2}}$ \\
\hline $\mathbf{S T}_{\mathbf{1}}$ & 0.132 & 0 & 0 & 0 \\
\hline $\mathbf{A T}_{\mathbf{1}}$ & sym & 1.001 & 0 & 0 \\
\hline $\mathbf{S T}_{\mathbf{2}}$ & sym & sym & 3.845 & 0 \\
\hline $\mathbf{A T}_{\mathbf{2}}$ & sym & sym & sym & 10.50 \\
\hline
\end{tabular}

\begin{tabular}{|c|c|c|c|c|}
\hline$\Delta \boldsymbol{T}=\mathbf{3 5}^{\circ} \mathbf{F}$ & $\mathbf{S T}_{\mathbf{1}}$ & $\mathbf{A T}_{\mathbf{1}}$ & $\mathbf{S T}_{\mathbf{2}}$ & $\mathbf{A T}_{\mathbf{2}}$ \\
\hline $\mathbf{S T}_{\mathbf{1}}$ & -0.543 & 0 & 0.534 & 0 \\
\hline $\mathbf{A T}_{\mathbf{1}}$ & sym & -1.524 & 0 & -0.940 \\
\hline $\mathbf{S T}_{\mathbf{2}}$ & sym & sym & -1.577 & 0 \\
\hline $\mathbf{A T}_{\mathbf{2}}$ & sym & sym & sym & 1.098 \\
\hline
\end{tabular}

\begin{tabular}{|c|c|c|c|c|}
\hline $\boldsymbol{\Delta} \boldsymbol{T}=\mathbf{7 0}^{\circ} \mathbf{F}$ & $\mathbf{S T}_{\mathbf{1}}$ & $\mathbf{A T}_{\mathbf{1}}$ & $\mathbf{S T}_{\mathbf{2}}$ & $\mathbf{A T}_{\mathbf{2}}$ \\
\hline $\mathbf{S T}_{\mathbf{1}}$ & -1.218 & 0 & 1.068 & 0 \\
\hline $\mathbf{A T}_{\mathbf{1}}$ & sym & -4.049 & 0 & -1.880 \\
\hline $\mathbf{S T}_{\mathbf{2}}$ & sym & sym & -6.998 & 0 \\
\hline $\mathbf{A T}_{\mathbf{2}}$ & sym & sym & sym & -8.303 \\
\hline
\end{tabular}


Another observation from Table 2 is that the linear stiffness coefficients vary linearly with temperature. Relative to the room temperature condition, the change in magnitude of a linear stiffness coefficient computed at a $\Delta T$ of $70^{\circ} \mathrm{F}$ is double that of the value computed at a $\Delta T$ of $35^{\circ} \mathrm{F}$. Again, this observation is consistent with the classic FE development ${ }^{13}$ and with previous indirect method work. ${ }^{9}$ Although this observation is not needed in the process of computing the set of modal stiffness coefficients by the indirect stiffness evaluation method, it may be exploited when temporal temperature variations are required in the analysis. Effectively, it allows a significant computational savings, since only a few linear modal coefficients must be scaled, but not reevaluated, as the temperature changes.

Finally, for the elevated temperature case, the change in sign of the affected diagonal terms from positive to negative provides useful information. Consider a basis consisting of only the first ST mode, so the linear stiffness corresponds to only the 1-1 entry in Table 2. A straight line fit at a function of temperature indicates a change in sign at $6.8^{\circ} \mathrm{F}$. Since the first ST mode greatly contributes to the first buckled shape, the temperature at which the sign changes is close to the first critical buckling temperature $\Delta T_{C R 1}$ of $6.6^{\circ} \mathrm{F}$. Similarly, a basis consisting of only the first AT mode shows a stiffness sign change at $13.9^{\circ} \mathrm{F}$, which is close to the second critical buckling temperature $\Delta T_{C R 2}$ of $13.6^{\circ} \mathrm{F}$. As the temperature increases, the number of negative diagonal entries increases. In Table 2 for example, there are three negative diagonal entries at a $\Delta T$ of $35^{\circ} \mathrm{F}$, and four negative diagonal entries at a $\Delta T$ of $70^{\circ} \mathrm{F}$.

\section{Dynamic Thermal Buckling Analysis}

The dynamic response during thermal buckling is of interest because it captures the characteristics of a single snap-through event. The dynamic thermal buckling event was induced by an instantaneous temperature increase of $35^{\circ} \mathrm{F}$, uniformly distributed along the length of the beam with a zero through-the-thickness gradient. The beam was initially at rest, with zero displacement and velocity. In the case of the reduced-order analysis, the initial conditions were specified in modal coordinates. A small decaying transverse perturbation force having an initial magnitude of 0.125-lbf was applied at the mid-span node to trigger the stability loss. The force linearly decayed to zero over the period of 0.5 s. A fixed integration time step of $1 \mu$ s was used for all reduced-order analyses.

\section{A. Physical DoF Analysis}

For comparison purposes, the physical DoF analysis was performed using ABAQUS/Explicit. The double precision explicit integration scheme with an automatically determined stable time integration step (referred to as "element by element" in ABAQUS) was utilized for all analyses. The finite element model used was identical to the MSC.NASTRAN-based model used in the RANSTEP reduced-order analysis, except that ABAQUS B21 beam elements were used in place of CBEAM elements.

\section{B. Thermal Buckling Response}

The displacement response of the mid-span node is shown in Figure 2 and Figure 3 for the transverse and inplane components, respectively. At this location, only the ST modes directly contribute to the transverse response, and only the SI modes directly contribute to the in-plane response. The effect of the AT and AI modes is therefore manifested indirectly by altering the system of modal equations. Consequently, modal time histories corresponding to modes ST and SI differ between the 12- and 24-mode bases. In Figure 2, the 24-mode RANSTEP and physical DoF analyses agree quite well, rising from the zero condition with a damped transient response about the final buckling displacement of 0.215-in. The 12-mode RANSTEP analysis comes to the same final displacement, but oscillates more toward the positive side of the final displacement. In Figure 3, it is interesting to note that significant in-plane response is not instantaneous, but is delayed by about $0.05 \mathrm{~s}$, clearly indicating the time-varying contribution of the in-plane modes. Since the 12-mode basis lacked the SI modes, this basis was incapable of representing any in-plane behavior. The damped transient response eventually settles to the expected zero in-plane displacement associated with the static buckled shape.

Note that a static post-buckling analysis performed with MSC.NASTRAN solution 106 (not shown) yielded nearly identical final buckling displacements. Hence, there are insignificant differences in the manner in which the thermal loading is handled between the NASTRAN-based RANSTEP analysis and the ABAQUS analysis. What is important about the dynamic thermal bucking analysis in this work, however, is not the final at-rest state, but the transient response leading to that state. In this light, the 24-mode basis best captures the relevant dynamics of the single snap-through event. Since the thermal-acoustic response next considered contains multiple snap-through events, it is expected that the 24-mode basis will also better represent the dynamics than the 12-mode basis. 


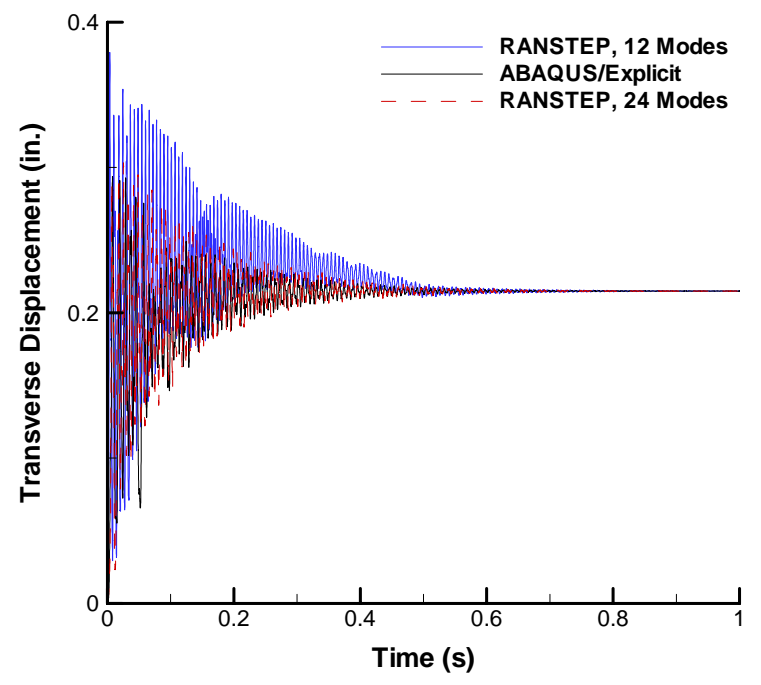

Figure 2: Transverse displacement response of a dynamic thermal buckling event.

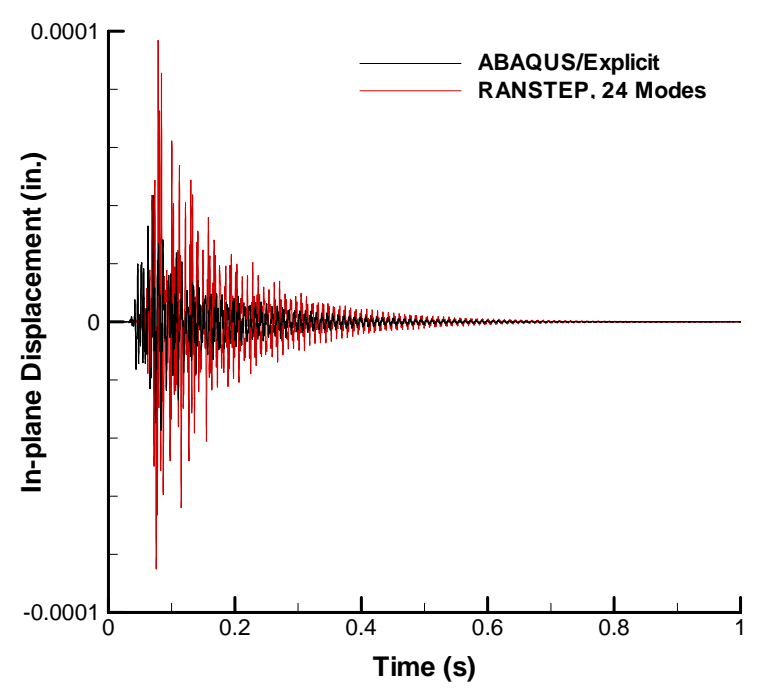

Figure 3: In-plane displacement response of a dynamic thermal buckling event.

\section{Thermal-Acoustic Response Analysis}

The beam response to a combined thermal-acoustic load was next investigated. The thermal load was instantaneously applied to the beam via a uniformly distributed temperature increase of $35^{\circ} \mathrm{F}$ with a zero throughthe-thickness gradient. A random pressure load with a flat excitation spectrum from 0-1500 Hz was generated using a previously developed procedure. ${ }^{14}$ The pressure was uniformly applied along the span in the transverse direction, irrespective of the deformation, i.e. follower forces were not utilized. Three pressure levels of $128 \mathrm{~dB}, 158 \mathrm{~dB}$, and $170 \mathrm{~dB}$ were considered so that different response regimes could be investigated.

The beam was initially at rest, with zero displacement and velocity, in the unbuckled state. A simulated response time history of 2.1384s was performed at each level considered. In the computation of power spectral density (PSD) and probability density function (PDF), five ensembles were averaged. For each ensemble, the initial $0.5 s$ was removed to eliminate the start-up transient.

\section{A. Thermal-Acoustic Response}

Three response regimes are illustrated with ABAQUS/Explicit-generated transverse displacement time histories of the mid-span node in Figure 4. The regimes can be characterized as: (i) small amplitude vibration around one of the two stable buckling equilibrium positions $(128 \mathrm{~dB})$, (ii) intermittent snap-through response between the two buckling equilibrium positions (158 dB), and (iii) persistent snap-through response between the two equilibrium positions $(170 \mathrm{~dB})$.

The PSD of the mid-span transverse displacement response at $128 \mathrm{~dB}$ is shown in Figure 5. Since the time histories from which these were generated lacked the initial thermal-buckling transient, the results from the 12- and 24-mode bases compare equally well with the physical DoF simulation. A significant static displacement component due to thermal buckling is apparent. The corresponding PDF is Gaussian, with a non-zero mean (not shown). With the initial thermal-buckling transient removed, the in-plane displacement response was negligible at this location.

When the level is increased such that intermittent snap-through response occurs, differences between the 12- and 24-mode bases become apparent. Figure 6 shows the PSD of the mid-span transverse displacement response at 158 $\mathrm{dB}$. Very good agreement is noted between the 24-mode and physical DOF analyses across the frequency range. The 12-mode reduced-order analysis however exhibits amplification at some peaks. All three analyses reflect the dominant zero-frequency component. The in-plane response is shown in Figure 7. Again, the 24-mode solution captured all of the essential features of the physical response. The 12-mode solution is unable to produce any inplane displacement response due to the lack of SI modes in the basis. 


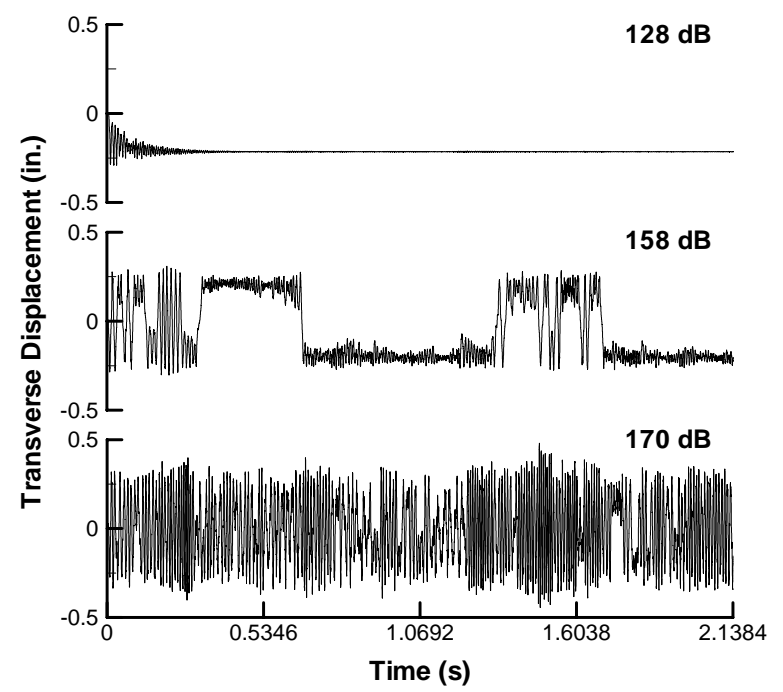

Figure 4: Transverse displacement response at $35^{\circ} \mathrm{F}$ and 128,158 , and $170 \mathrm{~dB}$ excitations.

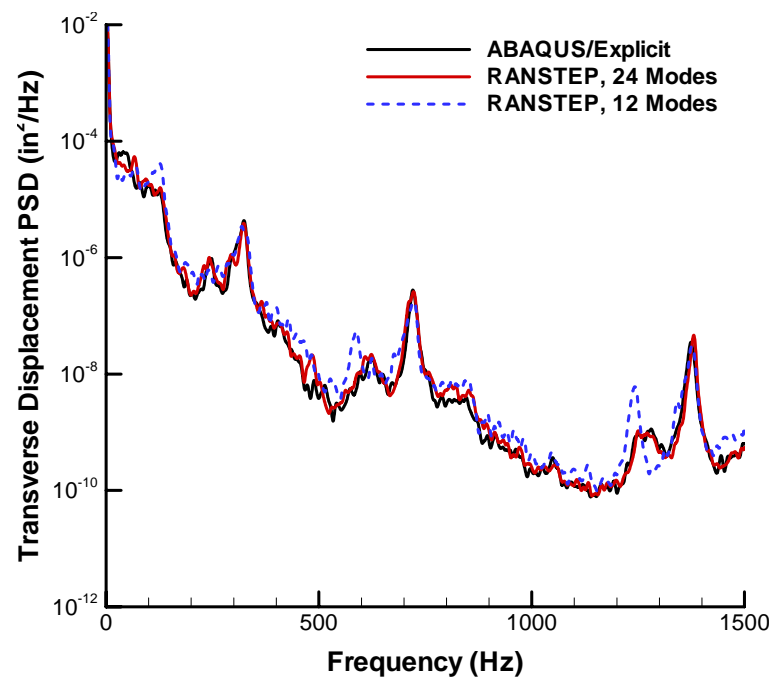

Figure 6: Mid-span transverse displacement response $\mathrm{PSD}$ at $35^{\circ} \mathrm{F}$ and $158 \mathrm{~dB}$.

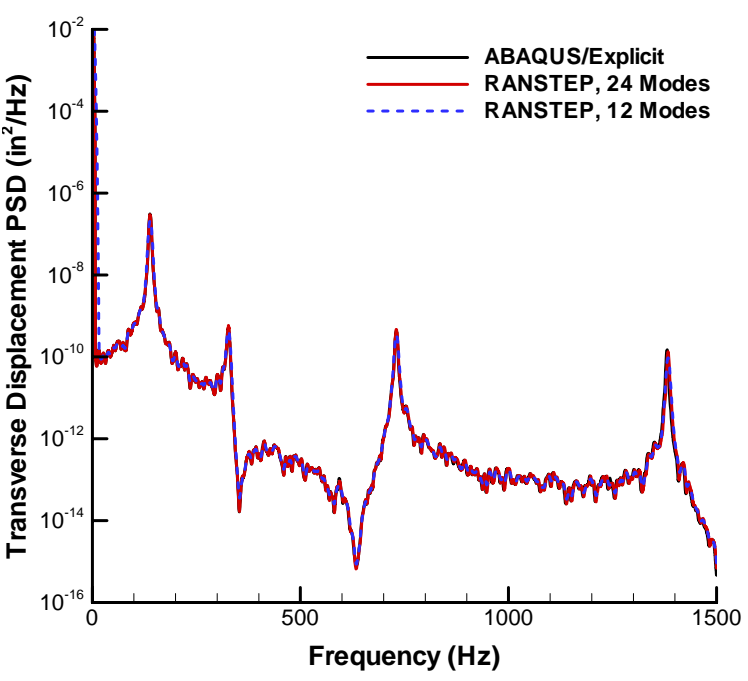

Figure 5: Mid-span transverse displacement response $\mathrm{PSD}$ at $35^{\circ} \mathrm{F}$ and $128 \mathrm{~dB}$.

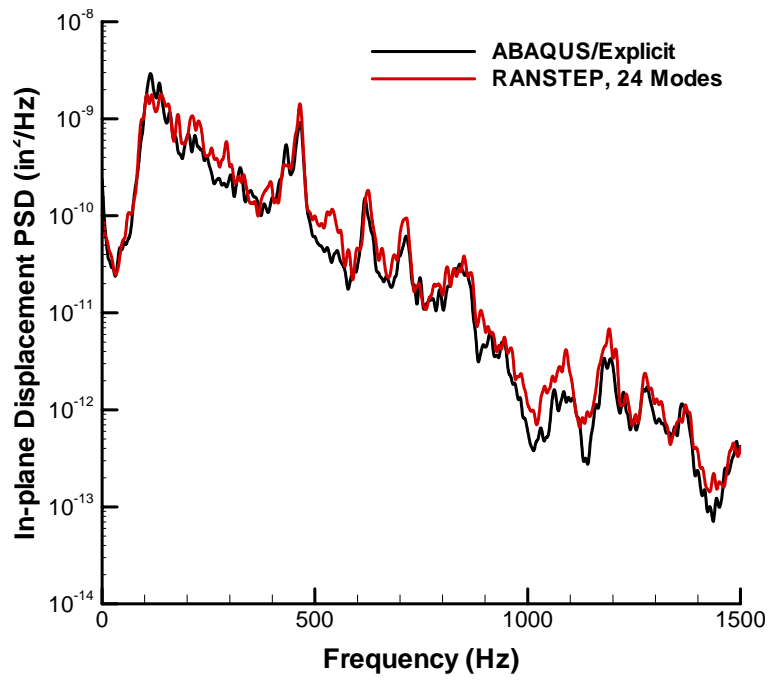

Figure 7: Mid-span in-plane displacement response PSD at $35^{\circ} \mathrm{F}$ and $158 \mathrm{~dB}$.

The transverse displacement response PDF at the mid-span is shown in Figure 8 for the physical DoF and 24mode reduced-order analyses. It is clear that the majority of vibration cycles at this level occurred about either of the two thermally buckled equilibrium positions. In particular, for the simulation period considered, the structure happened to spend more time oscillating about the lower equilibrium position. This is also consistent with the 158 $\mathrm{dB}$ time history response presented in Figure 4. Small values of the probability density in the proximity of the zero distribution range indicate the few instances when the response snapped between the two equilibrium positions.

It is often advantageous to observe the behavior at more than one location to help further identify limitations. The quarter-span location was selected for this purpose, as the displacement response there exhibits significant inplane and transverse components. The quarter-span transverse displacement response PSD at the $158 \mathrm{~dB}$ excitation level is shown in Figure 9. Like the mid-span response in Figure 6, the 24-mode solution compares favorably with the physical DoF solution across the frequency range. Above $1 \mathrm{kHz}, 12$-mode solution misses one peak and amplifies another. Again, the dominant zero-frequency component is seen. The quarter-span in-plane displacement PSD is shown in Figure 10. The 24-mode solution again compares favorably with the physical DoF solution across the frequency range, while the 12-mode solution poorly matched the physical response in the low and highfrequency ranges. 
To help better understand how these differences manifest themselves, one can observe the contribution of various modal displacement components. Plotted in Figure 11 are the lowest components of each mode type ( $\mathrm{ST}_{1}$, $\mathrm{AT}_{1}, \mathrm{SI}_{1}$, and $\mathrm{AI}_{1}$ ) for the 24-mode reduced-order solution at $35^{\circ} \mathrm{F}$ and $158 \mathrm{~dB}$. Also plotted are the reduced-order in-plane and transverse displacement time histories obtained from the inverse modal transformation at the quarterspan location. The modal responses from modes $\mathrm{ST}_{1}$ and $\mathrm{AT}_{1}$ are on the order of $10^{-3}$, while those from the $\mathrm{SI}_{1}$ and $\mathrm{AI}_{1}$ modes are two orders of magnitude smaller. Mode $\mathrm{ST}_{1}$ appears to be strongly correlated with the transverse physical displacement $(v)$, and mode $\mathrm{AI}_{1}$ appears to be strongly correlated with the in-plane physical displacement $(u)$. Of most interest in this figure are modes $\mathrm{SI}_{1}$ and $\mathrm{AT}_{1}$, which are not included in the 12-mode basis. These modes exhibit similar behavior to that of the decaying in-plane transient response of the single dynamic thermal buckling event, first seen in Figure 3. Unlike the mid-span location however, both modes directly contribute to the response at the quarter-span location. A significant increase in the $\mathrm{SI}_{1}$ and $\mathrm{AT}_{1}$ modal responses occur at every snap-through event. Hence these modes play an important role in the response during such events, and consequently indicate that the 12-mode basis is insufficient for accurately capturing the snap-through response.

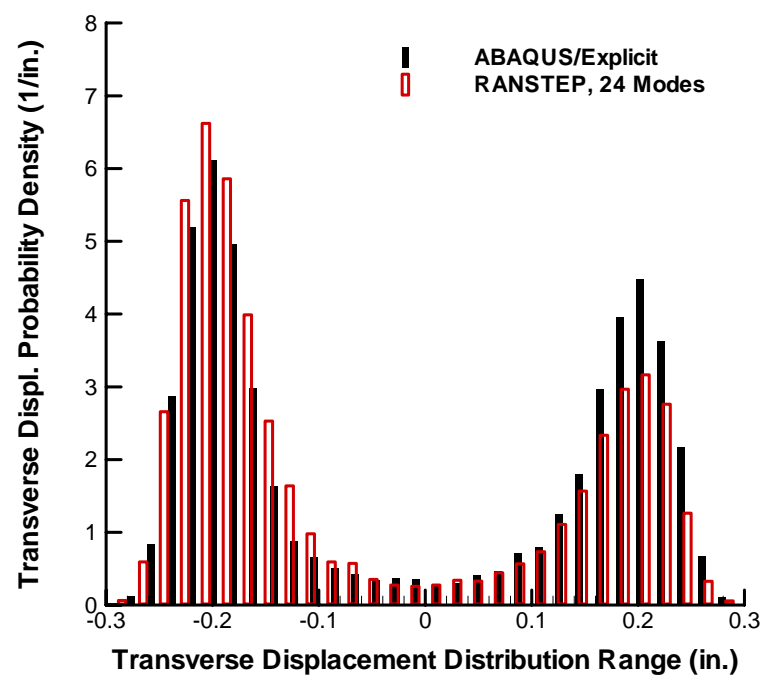

Figure 8: Mid-span transverse displacement response $\mathrm{PDF}$ at $35^{\circ} \mathrm{F}$ and $158 \mathrm{~dB}$.

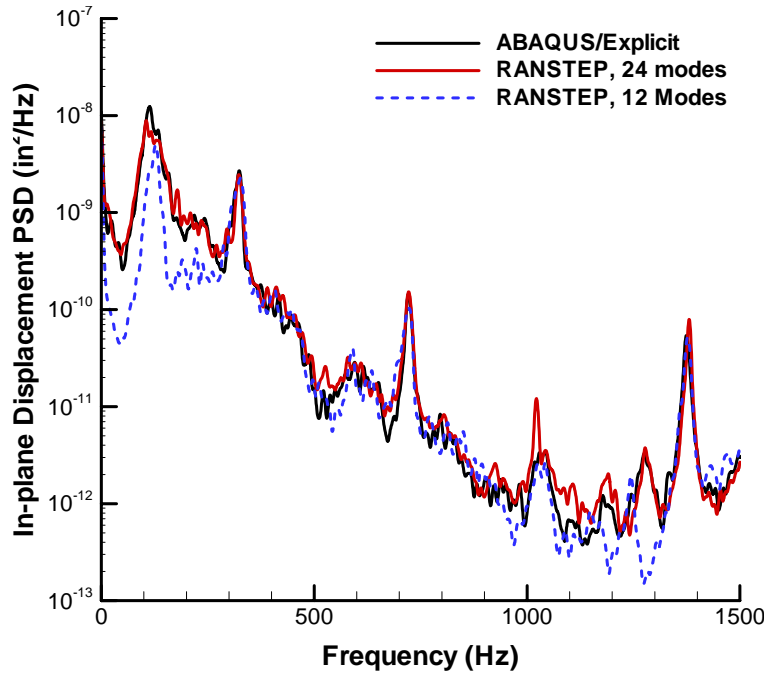

Figure 10: Quarter-span in-plane displacement response $\mathrm{PSD}$ at $35^{\circ} \mathrm{F}$ and $158 \mathrm{~dB}$.

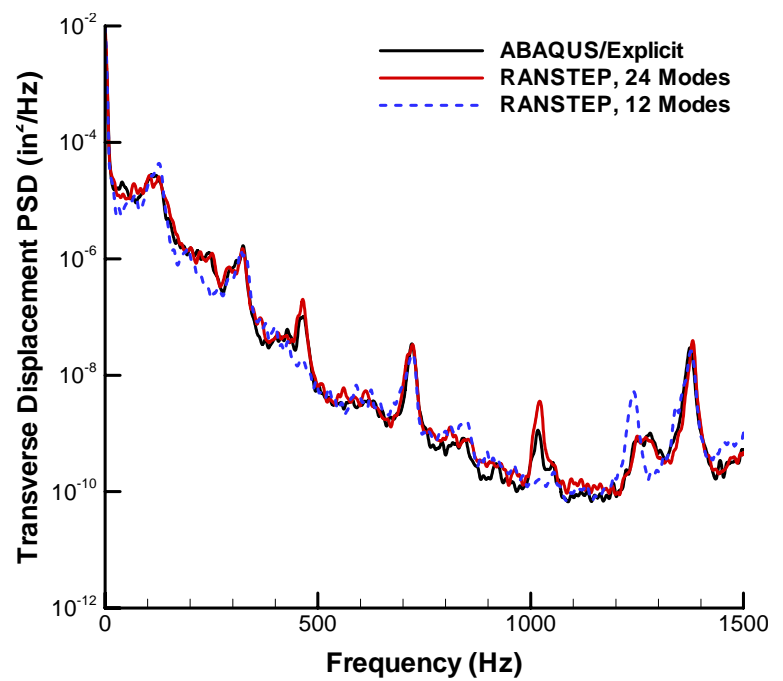

Figure 9: Quarter-span transverse displacement response $\mathrm{PSD}$ at $35^{\circ} \mathrm{F}$ and $158 \mathrm{~dB}$.

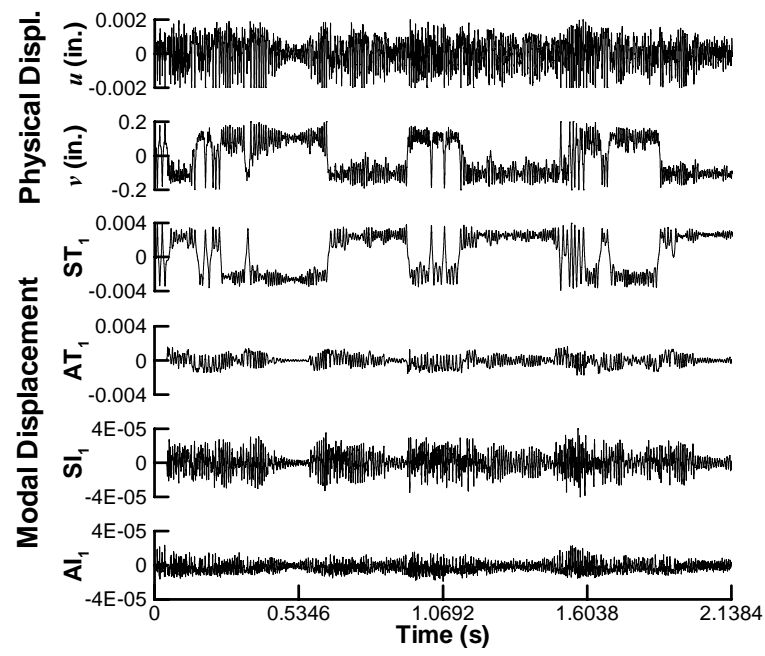

Figure 11: Quarter-span physical and modal displacement response at $35^{\circ} \mathrm{F}$ and $158 \mathrm{~dB}$.

The mid-span transverse and in-plane displacement response PSDs, obtained at a $\Delta T$ of $35^{\circ} \mathrm{F}$ and at the $170 \mathrm{~dB}$ excitation level, are presented in Figure 12 and Figure 13, respectively. This excitation was shown in Figure 4 to result in a persistent snap-through response. Both 24-mode PSDs compare very well with the physical DOF 
solution. As expected, the transverse response PSD from the 12-mode solution compares less favorably, and does not exist for the in-plane response. Because of the persistent snap-through response, the transverse static component was no longer dominant, as shown in Figure 12. The transverse displacement response PDF for this condition is shown in Figure 14. Here it is shown that as the intensity of snap-through is increased, the rate of the zero-crossings increases. Hence, the center part of the PDF distribution fills in compared to the intermittent snap-through conditions.

Finally, for a fixed temperature increment of $35^{\circ} \mathrm{F}$, the number of transverse displacement zero-crossings is presented as a function of the random pressure excitation level. It is seen in Figure 15 that snap-through does not occur up to a random pressure level of about $152 \mathrm{~dB}(0.1152 \mathrm{psi})$. Then, beginning in the range between $152 \mathrm{~dB}$ and $158 \mathrm{~dB}(0.2304 \mathrm{psi})$, the snap-through behavior is initiated and the number of zero-crossings starts growing as the excitation level is further increased. Results obtained in physical DoF and by the reduced-order analysis are in a good agreement despite a relatively short simulation time.

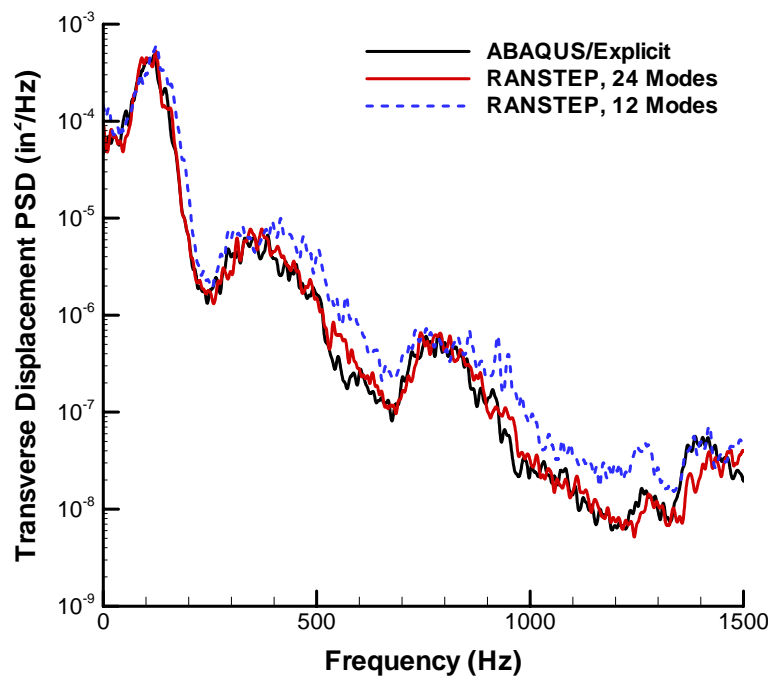

Figure 12: Mid-span transverse displacement response PSD response at $35^{\circ} \mathrm{F}$ and $170 \mathrm{~dB}$.

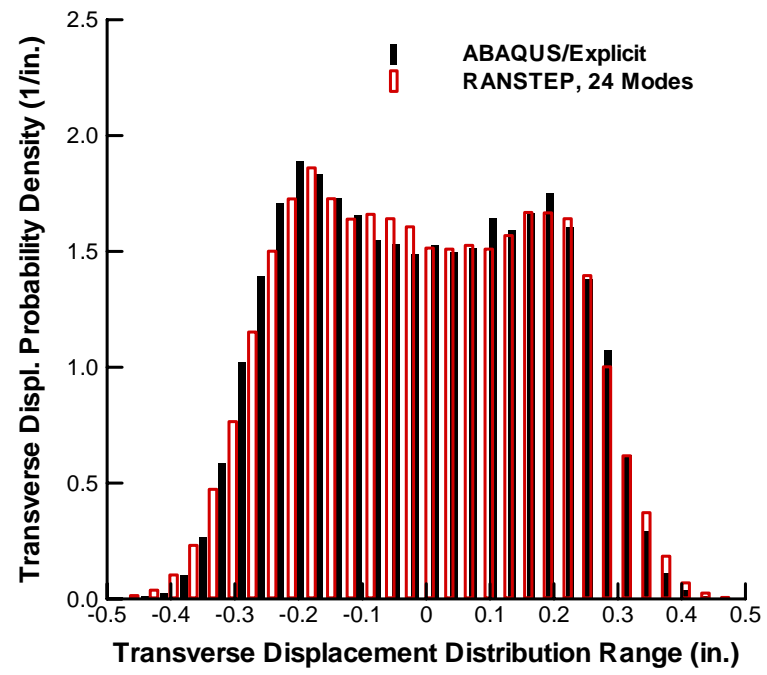

Figure 14: Mid-span transverse displacement response $\mathrm{PDF}$ at $35^{\circ} \mathrm{F}$ and $170 \mathrm{~dB}$.

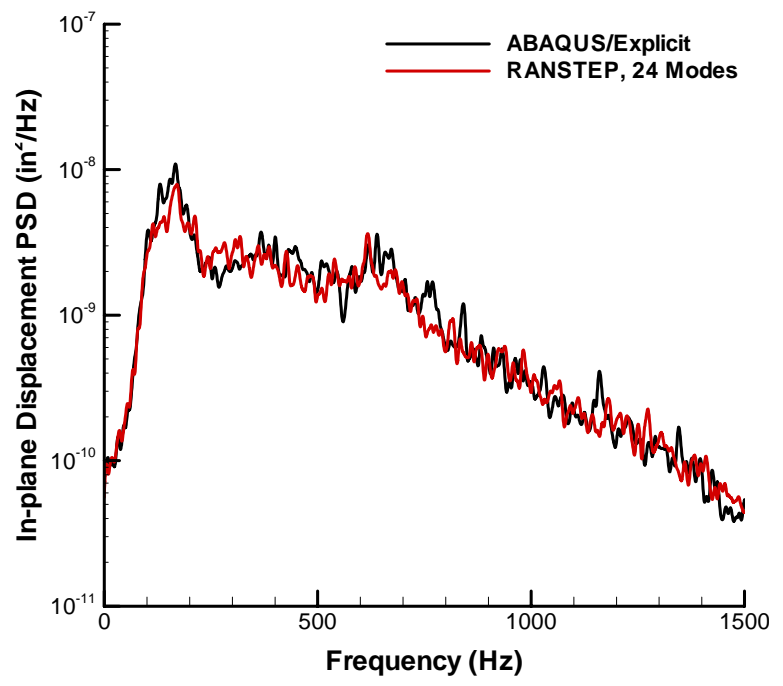

Figure 13: Mid-span in-plane displacement response PSD response at $35^{\circ} \mathrm{F}$ and $170 \mathrm{~dB}$.

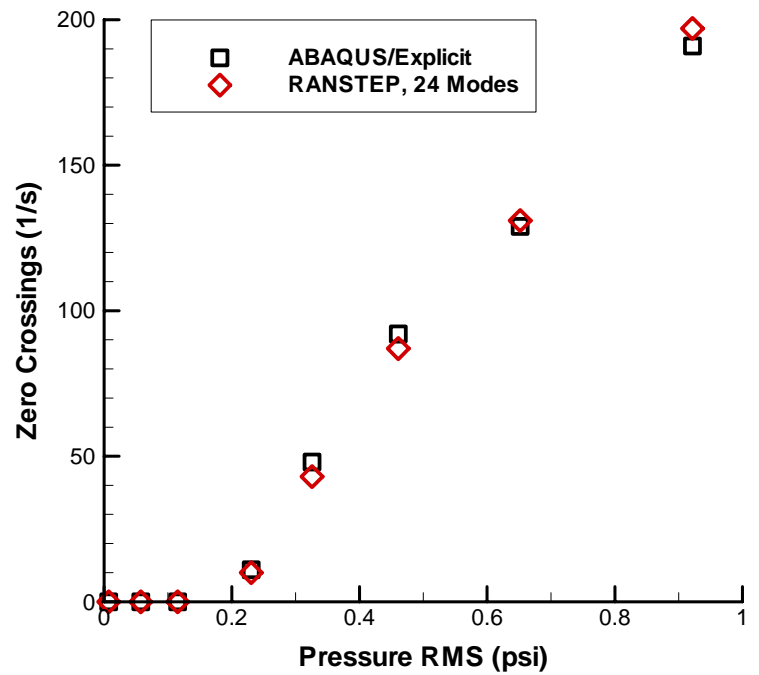

Figure 15: Transverse displacement zero-crossing intensity at $35^{\circ} \mathrm{F}$.

\section{Conclusion}

A reduced-order FE based method for predicting thermo-acoustic random response in a nonlinear regime was presented. Two sets of modal bases were examined in the study, and the corresponding reduced-order analysis results were compared with solutions obtained with an analysis in physical DoF. 
The effect of elevated temperature on the modal stiffness coefficients was first examined. It was found that only the linear stiffness coefficients corresponding to low-frequency transverse displacement modes were affected by the temperature change. These stiffness coefficients were found to vary linearly with temperature. Quadratic and cubic stiffness coefficients were unaffected. As a result, a computational benefit may be gained for problems with a timevarying thermal loading magnitude because linear coefficients need only be scaled.

In the analysis of dynamic thermal buckling and thermal-acoustic response, it was found that a modal basis consisting of four types of modes (ST, AT, SI and AI) more accurately predicted the response than a basis consisting of only ST and AI modes. In particular, for both loading conditions, the contribution of SI and AT modes becomes more significant as the structure transitions to a different equilibrium position.

Although not in scope of this study, the fatigue life will be affected in a different manner depending on the response regime. For the response about one of the thermally buckled equilibrium positions, a significant mean stress component will be introduced. The mean stress has been shown to adversely affect the fatigue life. ${ }^{15-17}$ Additionally, for intermittent and persistent snap-through, large cyclic stress amplitudes will rapidly accumulate and lead to a shorter fatigue life. Therefore, a continuation of this study to address stress recovery and fatigue estimation is deemed to be worthwhile.

\section{References}

${ }^{1} \mathrm{Ng}$, C.F. and Clevenson, S.A., "High intensity acoustic tests of a thermally stressed aluminum plate in TAFA," NASA TM 101552, 1989.

${ }^{2}$ Murphy, K.D., Virgin, L.N., and Rizzi, S.A., "Experimental snap-through boundaries for acoustically excited, thermally buckled plates," Experimental Mechanics, Vol. 36, No. 4, 1996, pp. 312-317.

${ }^{3}$ Istenes, R.R., Rizzi, S.A., and Wolfe, H.F., "Experimental nonlinear random vibration results of thermally buckled composite panels," Proceedings of the 36th AIAA/ASME/ASCE/AHS/ASC Structures, Structural Dynamics and Materials Conference, AIAA-95-1345-CP, New Orleans, LA, 1995, pp. 1559-1568.

${ }^{4}$ Lee, J., "Displacement and strain histograms of thermally buckled composite plates in random vibration," Proceedings of the 37th AIAA/ASME/ASCE/AHS/ASC Structures, Structural Dynamics, and Materials Conference, AIAA-96-1347, Salt Lake City, UT, 1996.

${ }^{5}$ Lee, J., "Displacement and strain statistics of thermally buckled plates," Journal of Aircraft, Vol. 38, No. 1, 2001, pp. 104-110.

${ }^{6}$ Lee, J., Vaicaitis, R., Wentz, K., Clay, C., Anselmo, E., and Crumbacher, R., "Predicition of statistical dynamics of thermally buckled composite plates," Proceedings of the 39th AIAA/ASME/ASCE/AHS/ASC Structures, Structural Dynamics, and Materials Conference, AIAA-98-1975, Long Beach, CA, 1998.

${ }^{7}$ Guo, X., Przekop, A., and Mei, C., "Nonlinear random response of shallow shells at elevated temperatures using finite element modal method," Proceedings of the 45the AIAA/ASME/ASCE/AHS/ASC Structures, Structural Dynamics and Materials Conference, AIAA-2004-1558, Palm Springs, CA, 2004.

${ }^{8}$ Mei, C., Dhainaut, J.M., Duan, B., Spottswood, S.M., and Wolfe, H.F., "Nonlinear random response of composite panels in an elevated thermal environment," Air Force Research Laboratory AFRL-VA-WP-TR-2000-3049, Wright-Patterson Air Force Base, OH, October 2000.

${ }^{9}$ Mignolet, M.P., Radu, A.G., and Gao, X., "Validation of reduced order modeling for the prediction of the response and fatigue life of panels subjected to thermo-acoustic effects," Structural Dynamics: Recent Advances, Proceedings of the 8th International Conference, Southampton, UK, 2003, M.J. Brennan, et al. (ed.).

${ }^{10}$ Muravyov, A.A. and Rizzi, S.A., "Determination of nonlinear stiffness with application to random vibration of geometrically nonlinear structures," Computers and Structures, Vol. 81, No. 15, 2003, pp. 1513-1523.

${ }^{11}$ Rizzi, S.A. and Przekop, A., "The effect of basis selection on static and random response prediction using nonlinear modal simulation," NASA TP-2005-213943, December 2005.

${ }^{12}$ Przekop, A. and Rizzi, S.A., "Nonlinear reduced order random response analysis of structures with shallow curvature," To appear in the AIAA Journal, 2006.

${ }^{13}$ Przekop, A., "Nonlinear response and fatigue estimation of aerospace curved surface panels to combined acoustic and thermal loads," Ph.D. Dissertation, Old Dominion University, Norfolk, VA, 2003.

${ }^{14}$ Rizzi, S.A. and Muravyov, A.A., "Comparison of nonlinear random response using equivalent linearization and numerical simulation," Structural Dynamics: Recent Advances, Proceedings of the 7th International Conference, Vol. 2, Southampton, UK, 2000, N.S. Ferguson, et al. (ed.), pp. 833-846.

${ }^{15}$ Sweitzer, K.A. and Ferguson, N.S., "Mean stress effects on random fatigue of nonlinear structures," XII International Congress on Sound and Vibration, Lisbon, Portugal, 2005.

${ }^{16}$ Dowling, N.E., "Mean stress effects in stress-life and strain-life fatigue," Fatigue 2004: Second SAE Brasil Conference on Fatigue, SAE 2004-01-2227, São Paulo, Brasil, 2004.

${ }^{17}$ Rizzi, S.A. and Przekop, A., "Estimation of sonic fatigue by reduced-order finite element based analyses," Structural Dynamics: Recent Advances, Proceedings of the 9th International Conference, Southampton, UK, 2006, M.J. Brennan, et al. (ed.). 